\title{
AVALIAÇÃO DA QUALIDADE DAS PRESCRIÇÕES DE ENFERMAGEM EM HOSPITAIS DE ENSINO PÚBLICO
}

Gelena Lucinéia Gomes da Silva VERSA ${ }^{\mathrm{b}}$, Ana Yassuko MURASSAKI ${ }^{\mathrm{c}}$, Larissa Gutierrez da SILVA ${ }^{\mathrm{d}}$, Dagmar Willamowius VITURI ${ }^{\mathrm{e}}$, Willian Augusto de MELLO ${ }^{\mathrm{f}}$, Laura Misue MATSUDA ${ }^{\mathrm{g}}$

\section{RESUMO}

Estudo multicêntrico, transversal, realizado entre dezembro de 2009 e junho de 2010, que teve como objetivo avaliar a qualidade das Prescrições de Enfermagem (PE) em dois hospitais de ensino público. A amostra se constituiu de 1.307 PE, e os dados foram tratados por meio dos testes G e Qui-quadrado. A determinação da qualidade das PE se baseou nos Índices de Classificação abordados na literatura. Dentre os resultados foram obtidas 1.083 (82,8\%) PE corretas e adequadas, 154 (11,8\%) inadequadas e 52 (3,9\%) incompletas. Em 18 (1,37\%) prontuários, as PE eram inexistentes. Houve significância estatística $(\alpha<0,05)$ para PE incompletas e ausentes entre os dois hospitais $(0,00)$, assim como PE inadequadas entre as faixas etárias (0,03). Conclui-se que, nos hospitais investigados, a PE necessita ser melhorada, seja em termos de quantidade como de qualidade.

Descritores: Processos de enfermagem. Avaliação em enfermagem. Qualidade da assistência à saúde.

\section{RESUMEN}

Estudio multicéntrico, transversal, realizado entre diciembre de 2009 a junio de 2010 y que tuvo como objetivo evaluar la calidad de Prescripción Enfermera (PE) en dos hospitales de enseñanza pública. La muestra consistió en 1307 PE y los datos fueron procesados a través de las pruebas estadísticas de G y chi-cuadrado. La determinación de la calidad de la PE se basó en los indices de clasificación reportados en la literatura. Entre los resultados obtenidos fueron 1.083 (82,8\%) PE correctas y adecuadas, 154 (11,8\%) inadecuadas y 52 (3,9\%) incompletas. En 18 (1,37\%) registros médicos, las PE no existían. Hubo significación estadística $(\alpha<0,05)$ para PE incompletas y ausentes entre los dos hospitales $(0,00)$ así como las PE inadecuadas entre grupos de edad (O,O3). Se concluye que en los hospitales consultados la PE debe mejorarse, tanto en términos de cantidad como de calidad.

Descriptores: Procesos de enfermería. Evaluación de enfermería. Calidad de la atención de salud. Título: Evaluación de la calidad de las prescripciones enfermeras en hospitales de enseñanza pública.

\section{ABSTRACT}

A multicenter, cross-sectional study took place from December 2009 to June 2010 and aimed to assess the quality of Nursing Prescription (NP) in two public teaching hospitals. The sample consisted of 1,307 NP and data were processed using the $G$-test and chi-square. The determination of the quality of NP was based on the classification indices reported in literature. Among the results, 1,083 (82.8\%) correct and appropriate NP, 154 (11.8\%) inadequate NP and 52 (3.9\%) incomplete NP were found. In $18(1.37 \%)$ patient charts, the NP was nonexistent. There was statistic significance $(\alpha<0.05)$ for incomplete and absent NP between the two hospitals (O.00), as well as inappropriate NP between age groups (0.03). It was concluded that, in the hospitals under study, the NP needs to be improved, both in terms of quantity and quality.

Descriptors: Nursing process. Nursing assessment. Quality of health care.

Title: Assessment of quality of nursing prescriptions in public teaching hospital.

a Produto do Projeto de Pesquisa: Gestão em Saúde: Perspectivas da Qualidade. Financiado pelo CNPq. Edital Universal 14/2008.

b Enfermeira Administrativa do Hospital Universitário do Oeste do Paraná (HUOP). Mestre em Enfermagem pela Universidade Estadual de Maringá (UEM). Cascavel-Paraná (PR). Brasil.

c Enfermeira. Mestranda em Enfermagem na UEM. Maringá-PR. Brasil

d Enfermeira. Mestre em Enfermagem pela UEM. Londrina-PR. Brasil.

e Enfermeira do Hospital Universitário de Londrina - HUL - PR. Doutoranda em Enfermagem na Escola de Enfermagem de Ribeirão Preto. Londrina-PR. Brasil.

f Enfermeiro. Mestre em enfermagem. Docente do Centro Universitário de Maringá (Cesumar). Maringá-PR. Brasil.

g Enfermeira. Doutora em Enfermagem. Docente do Departamento de Enfermagem da UEM. Maringá-PR. Brasil. 


\section{INTRODUÇÃO}

Na contemporaneidade, a preocupação com os padrões de qualidade do atendimento ocorreu pelo avanço do capitalismo e pela crescente concorrência dos produtos e serviços. Tal situação de mudanças na economia ocasionou exigências no controle, na manutenção e na avaliação da qualidade dos serviços, sendo incluído neste contexto o setor da saúde ${ }^{(1)}$.

No que tange à avaliação em saúde como atividade indispensável ao alcance da qualidade, faz-se necessário a análise da estrutura, do processo e dos resultados dos serviços, com objetivo de determinar o grau de sucesso das ações desempenhadas ${ }^{(2)}$ de tal forma que desvios em relação aos padrões e erros possam ser precocemente detectados e corrigidos.

A identificação e a correção dos erros concernentes às atividades executadas pelos profissionais propiciam a otimização da qualidade dos cuidados $\operatorname{prestados}^{(3)}$. Dessa forma, a avaliação favorece a identificação das inconformidades no atendimento à saúde e possibilita readequações no serviço, de forma que o cuidado atenda às necessidades e expectativas dos clientes e consequentemente, tenha qualidade ${ }^{(4)}$.

No âmbito da enfermagem, o método que propicia o planejamento e a execução de cuidados sistematizados é o Processo de Enfermagem ${ }^{(5)}$ que se apresenta como ferramenta necessária à execução de cuidados de qualidade, visto que orienta as ações do enfermeiro e da sua equipe na tomada de decisão e no atendimento integral e individualizado ao cliente ${ }^{(6)}$.

No Brasil, o Processo de Enfermagem mais conhecido e utilizado foi proposto em 1960 por Wanda de Aguiar Horta, que estabeleceu ações sistematizadas para a assistência de enfermagem por meio de seis passos ou etapas: Histórico de Enfermagem; Diagnóstico de Enfermagem (DE); Plano Assistencial; Prescrição de Enfermagem (PE); Evolução e Prognóstico de Enfermagem ${ }^{(7)}$.

Na utilização do Processo de Enfermagem, as necessidades do cliente são identificadas pelo Diagnóstico de Enfermagem e atendidas/realizadas por meio da PE que possibilita o planejamento e a execução do cuidado ${ }^{(5)}$. Desse modo, a PE se destaca como um meio essencial para o atendimento holístico aos pacientes e também para a gestão do cuidado, visto que subsidia o planejamento, a orientação, a execução e a avaliação dos cuidados ${ }^{(7)}$, atuando como um dos indicadores do trabalho da enfermagem por permitir, mesmo de forma indireta, a avaliação de como o cuidado foi planejado e executado(6).

No tocante aos registros/anotações oriundas do PE em geral, sabe-se que existe uma correlação positiva entre o registro e o cuidado prestado. Desta forma, por meio da documentação e registros de todas as ações de enfermagem, é possível avaliar a qualidade dos cuidados prestados ${ }^{(3)}$.

Quanto ao registro na PE, este é um dos melhores instrumentos de comunicação da enfermagem, pois permite a transmissão efetiva de informações entre os membros da equipe multiprofissional; possibilita o re-planejamento dos cuidados; à avaliação da qualidade da assistência e; a comprovação legal para o paciente, profissional e à instituição, em casos de processos éticos ${ }^{(3,8-9)}$.

Ao considerar a PE como norteadora do processo de assistência e instrumento de manutenção e/ou avaliação da qualidade do cuidado de enferma$\mathrm{gem}^{(6)}$ se faz necessário que o enfermeiro a realize com rigor técnico-científico ${ }^{(10)}$, de modo a planejar cuidados que promovam a recuperação da saúde do paciente/cliente/usuário ${ }^{(11)}$. Nessa perspectiva, a Prescrição elaborada de forma individualizada, com base em conhecimento técnico e científico, tende a resultar em cuidados de qualidade ${ }^{(6)}$.

No âmbito da orientação/determinação do cuidado, apesar da importância da PE no processo de atendimento da enfermagem, observa-se que no Brasil, essa etapa ainda não é realizada rotineiramente e, quando ocorre, em geral não apresenta algumas características fundamentais como: exatidão; clareza; confiabilidade; validade e coerência com as necessidades dos pacientes/clientes ${ }^{(12)}$.

Cabe ressaltar que o direcionamento contínuo das ações de enfermagem por meio da PE, não apenas em situações pontuais, permite que as necessidades individuais dos pacientes, que são passíveis de se alterarem a qualquer momento, sejam atendidas de forma específicas e seguras.

Estudos brasileiros a respeito da elaboração e utilização da PE apontam, dentre outras dificuldades, o despreparo do enfermeiro; o pouco interesse em realizá-la ${ }^{(13,14)}$; insuficiência de conhecimentos teóricos básicos e específicos sobre a metodologia; e em especial, dificuldades com a identificação dos Diagnósticos de Enfermagem que fundamentam a elaboração da $\mathrm{PE}^{(14,15)}$.

No cotidiano da prática na enfermagem, tem-se observado que as dificuldades antes referidas também estão presentes e com isso, não raras vezes, 
o enfermeiro se apresenta inseguro, desmotivado e, até mesmo resistente e desacreditado em realizar integralmente o Processo de Enfermagem.

Com o intuito de obter informações que contribuam à qualidade da assistência de enfermagem, a realização deste estudo se justifica porque o conhecimento de fatores que intervém positiva ou negativamente na elaboração da PE é uma ferramenta importante, não apenas para o diagnóstico situacional do setor/serviço, mas também para nortear o desenvolvimento de ações educativas, que promovam a realização correta da $\mathrm{PE}$.

Com base no exposto, intentando contribuir com o aporte de conhecimento acerca do tema, questiona-se: A Prescrição de Enfermagem de dois hospitais de ensino público, atende aos quesitos de qualidade enunciados por Manganaro ${ }^{(16)}$ Para responder a essa questão, se propõe a realização deste estudo que tem como objetivo avaliar a qualidade das Prescrições de Enfermagem de dois hospitais de ensino públicos.

\section{MÉTODOS}

Estudo multicêntrico e transversal, realizado em dois hospitais universitário públicos, Hospital 1 e Hospital 2, situados no estado do Paraná, os quais dispõem de 30 e 121 leitos destinados à Clínica Médica e Clínica Cirúrgica, respectivamente.

Para a composição da população do estudo, consideraram-se os seguintes critérios de inclusão: estar internado no mínimo há 24 horas, em uma das unidades investigadas (Clínica Médica (CM); Clínica Cirúrgica (CC), do Hospital 1; Unidade Clínica e Cirúrgica Feminina (UF); Unidade Clínica e Cirúrgica Masculina (UM), do Hospital 2 e aceitar formalmente em participar do estudo.

Todos os pacientes internados, que atenderam aos critérios de inclusão, foram avaliados, assim como sua respectiva $\mathrm{PE}$, do dia anterior à visita do avaliador. O censo, tipo de amostra do estudo, de todos os sujeitos de pesquisa resultou em 1307 observações do cliente e seu respectivo prontuário. No entanto, 18 pacientes não tinham a PE do dia anterior, objeto de avaliação. Dessa forma, foram avaliados 1307 pacientes e $1289 \mathrm{PE}$. Do total de PE avaliadas, 374 foram do hospital 1 e 933 do hospital 2.

A coleta de dados ocorreu nos meses de fevereiro, março e abril de 2010, em seis dias aleatórios de cada mês, por meio de observação direta do paciente e sua PE do dia anterior, utilizando-se do "Instrumento de Registro de Busca Ativa", composto por 52 questões, elaborado e validado por Vituri(17).

Neste estudo, em razão da sua temática, somente quatro assertivas do referido instrumento foram abordadas: 1. Número de pacientes com PE diária adequada às suas necessidades; 2. Número de pacientes com PE diária incompleta em relação às suas necessidades; 3. Número de pacientes com PE diária inadequada, ou seja, com cuidados prescritos que não dizem respeito às suas necessidades atuais e; 4. Número de pacientes com impresso de PE, porém SEM cuidados prescritos pelo enfermeiro.

Vale lembrar que foram analisadas as PE das últimas 24 horas de internamento do paciente na unidade. Com isso, categorizou-se como Prescrição "não preenchida" a ausência de PE nesse período.

$\mathrm{O}$ instrumento foi preenchido pelos pesquisadores, após avaliação do paciente e de sua PE no que tange ao atendimento aos critérios para sua classificação como: "Adequada" ou "Inadequada" às necessidades do paciente e; "Completa" ou "Incompleta” em relação aos padrões técnicos da PE.

A análise das PE foi realizada com base na abordagem de Manganaro ${ }^{(16)}$ que estabelece padrões técnicos e características básicas para a PE, quais sejam: ser precedida de data; conter a determinação dos horários e de como o cuidado deve ser executado; ser elaborada diariamente para um período de 24 horas; estar condizente com as necessidades do paciente; incluir a verificação dos sinais vitais pelo menos uma vez ao dia e; estar assinada pelo enfermeiro que a elaborou.

Para a classificação da qualidade das PE, utilizou-se como padrão os Índices de Classificação sugeridos no estudo de Cianciarullo, Fugulin e Andreoni $^{(18)}$. A referida classificação determina que uma PE de qualidade necessita apresentar acima de $80 \%$ dos itens "Preenchidos Completamente"; até $15 \%$ de "Preenchimento Incompleto"; menos de 5\% para itens da Prescrição "Não Preenchidos" e 0\% para itens "Incorretos". Desse modo, neste estudo as PE serão consideradas de Qualidade se os dados corresponderem aos parâmetros citados.

No tratamento dos dados, utilizou-se o Programa Excel e a seguir, foram realizadas análises estatísticas por meio dos softwares Statistic 8.0 e Bioestat 5.0, constituída pelos testes de Independência $G$ para as variáveis que apresentavam tabela de contingência $4 \times 2$ e o teste Qui-quadrado 
para as tabelas com variáveis dicotômicas ou com tabela de contingência $2 \times 2$. Em todos os testes considerou-se intervalo de confiança de $95 \%$ e nível de significância de 5\%.

Este estudo obedeceu todas as exigências da Resolução 196/1996 ${ }^{(19)}$ e o parecer favorável à sua realização foi emitido pelo Comitê Permanente de Ética em Pesquisas com Seres Humanos (COPEP) da Universidade Estadual de Maringá-PR, sob o $n^{0}$. 482/2009 e do Comitê de Ética em Pesquisas Envolvendo Seres Humanos (CEP) da Universidade Estadual de Londrina - PR, sob o nº. 252/2009.

\section{RESULTADOS}

Foram avaliados 1307 prontuários e encontrou-se 1289 (98,6\%) PE. A maioria era de pacientes do sexo masculino 702 (53,7\%), com média de idade de 52,6 anos $(\sigma=19,13)$ e a faixa etária mais prevalente de 35 a 59 anos $(44,7 \%)$.

Na Tabela 1 constam os dados das PE analisadas quanto aos padrões de adequação às necessidades dos pacientes e aos quesitos básicos e técnicos necessários às Prescrições, como data, assinatura e aprazamento.

Na Tabela 2 constam os dados referentes aos prontuários que apresentaram ou não a PE.
Na Tabela 3, os padrões de qualidade à classificação das $\mathrm{PE}$, correspondem à abordagem de Cianciarullo, Fugulin e Andreoni ${ }^{(18)}$.

\section{DISCUSSÃO}

$\mathrm{Na}$ análise da adequação das $\mathrm{PE}$ às necessidades dos pacientes (Tabela 1), as duas instituições (Hospital 1 e 2 ) apresentaram prevalência de cuidados prescritos inadequadamente (12,9\% e $11,5 \%$ respectivamente), sem diferença significativa entre esses hospitais $(\alpha=0,56)$, o que aponta à existência de um problema em comum. Esse dado é preocupante porque, uma $\mathrm{PE}$ incompatível com as necessidades do paciente consiste em ameaça à sua segurança e por isso, a sua elaboração deve estar pautada em padrões de qualidade, sem a presença de erros e/ou inadequações ${ }^{(5)}$.

Em análise da faixa etária encontrou-se diferença estatística $(\alpha=0,03)$ quanto às prescrições inadequadas para esse grupo, pois as taxas de PE inadequadas para os pacientes jovens $(<20$ anos) e adultos (20-34 anos) foram superiores às outras faixas e essa diferença pode estar relacionada à prática de estabelecer prioridade aos grupos de maiores riscos, privilegiando-se os cuidados prioritários aos pacientes mais debilitados, como os idosos ${ }^{(7)}$. Destarte, apesar de não se ter encontrado nenhuma publicação

Tabela 1: Distribuição das Prescrições de Enfermagem $(n=1289)$ de acordo com a adequação às necessidades dos pacientes e aos padrões básicos para elaboração, segundo a variável idade e local de internação. Maringá-PR, 2010.

\begin{tabular}{|c|c|c|c|c|c|c|c|c|c|c|c|}
\hline \multirow[b]{2}{*}{ Dados } & \multicolumn{2}{|c|}{$\begin{array}{c}\text { Prescrição } \\
\text { adequada }\end{array}$} & \multicolumn{2}{|c|}{$\begin{array}{l}\text { Prescrição } \\
\text { inadequada }\end{array}$} & \multirow[b]{2}{*}{$\alpha$} & \multirow[b]{2}{*}{ Dados } & \multicolumn{2}{|c|}{$\begin{array}{c}\text { Prescrição } \\
\text { completa }\end{array}$} & \multicolumn{3}{|c|}{$\begin{array}{l}\text { Prescrição } \\
\text { incompleta }\end{array}$} \\
\hline & $\mathbf{n}$ & $\%$ & $\mathbf{n}$ & $\%$ & & & $\mathbf{n}$ & $\%$ & $\mathbf{n}$ & $\%$ & $\alpha$ \\
\hline Hosp. & & & & & 0,56 & Hosp. & & & & & $0,00^{4}$ \\
\hline Hosp. 1 & 317 & 87,8 & 047 & 12,9 & & Hosp. 1 & 329 & 90,3 & 035 & 9,61 & \\
\hline Hosp.2 & 818 & 88,4 & 107 & 11,5 & & Hosp.2 & 908 & 98,1 & 017 & 1,83 & \\
\hline Idade & & & & & $0,03^{*}$ & Idade & & & & & 0,34 \\
\hline$<20$ & 043 & 82,6 & 009 & 17,3 & & $<20$ & 047 & 03,6 & 005 & 0,38 & \\
\hline $20-34$ & 166 & 82,5 & 035 & 17,4 & & $20-34$ & 193 & 14,9 & 008 & 0,62 & \\
\hline $35-59$ & 519 & 90,1 & 057 & 09,9 & & $35-59$ & 555 & 43,0 & 021 & 1,69 & \\
\hline$>60$ & 407 & 88,4 & 053 & 11,5 & & $>60$ & 442 & 34,2 & 018 & 1,39 & \\
\hline
\end{tabular}

Fonte: Hospitais Universitários do Norte e Noroeste do Paraná, 2010. 
Tabela 2: Distribuição dos prontuários $(n=1307)$, de acordo com a presença ou ausência de Prescrição de Enfermagem, segundo a variável idade e local de internação. Maringá-PR, 2010.

\begin{tabular}{|c|c|c|c|c|c|c|c|c|c|c|c|}
\hline \multirow[b]{2}{*}{ Dados } & \multicolumn{2}{|c|}{$\begin{array}{l}\text { Prescrição } \\
\text { presente }\end{array}$} & \multicolumn{2}{|c|}{$\begin{array}{l}\text { Prescrição } \\
\text { ausente }\end{array}$} & \multirow[b]{2}{*}{$\alpha$} & \multirow[b]{2}{*}{ Dados } & \multicolumn{2}{|c|}{$\begin{array}{l}\text { Prescrição } \\
\text { presente }\end{array}$} & \multicolumn{2}{|c|}{$\begin{array}{l}\text { Prescrição } \\
\text { ausente }\end{array}$} & \multirow[b]{2}{*}{$\alpha$} \\
\hline & $\mathbf{n}$ & $\%$ & $\mathbf{n}$ & $\%$ & & & $\mathbf{n}$ & $\%$ & $\mathbf{n}$ & $\%$ & \\
\hline Idade & & & & & 0,53 & Hosp. & & & & & $0,02 *$ \\
\hline$<20$ & 052 & 96,3 & $\mathrm{O} 2$ & 3,70 & & Hosp 1 & 364 & 97,3 & 10 & 2,67 & \\
\hline $20-34$ & 201 & 99,0 & $\mathrm{O} 2$ & 0,98 & & Hosp 2 & 925 & 99,1 & 08 & 0,85 & \\
\hline $35-59$ & 576 & 98,4 & 09 & 1,53 & & & & & & & \\
\hline$>60$ & 460 & 98,9 & 05 & 1,07 & & & & & & & \\
\hline
\end{tabular}

Fonte: Hospitais Universitários do Norte e Noroeste do Paraná, 2010.

$\alpha=$ Dado representativo da diferença entre as variáveis.

* Nível descritivo para o Teste Qui-quadrado com correção de Yates que apresentaram significância estatística $(\alpha<0,05)$.

Tabela 3: Distribuição das coletas $(n=1307)$, segundo padrões de qualidade das Prescrições de Enfermagem. Maringá-PR, 2010.

\section{Padrão de qualidade das Prescrições de Enfermagem*}

\begin{tabular}{|c|c|c|c|}
\hline \multicolumn{4}{|c|}{ Instituições } \\
\hline \multicolumn{2}{|c|}{$\begin{array}{l}\text { Hospital } 1 \\
n=374\end{array}$} & \multicolumn{2}{|c|}{$\begin{array}{l}\text { Hospital } 2 \\
\text { n=933 }\end{array}$} \\
\hline n & $\%$ & $\mathbf{n}$ & $\%$ \\
\hline 282 & 75,4 & 801 & 85,85 \\
\hline 35 & 9,35 & 17 & 01,82 \\
\hline 10 & 2,67 & 08 & 00,85 \\
\hline 47 & 12,56 & 107 & 11,46 \\
\hline
\end{tabular}

Fonte: Hospitais Universitários do Norte e Noroeste do Paraná, 2010.

* Índices de Classificação das Prescrições de Cianciarullo, Fugulin e Andreoni ${ }^{(18)}$.

que aborde a inadequação de PE nos grupos jovens e adultos, deduz-se que isso pode ter ocorrido por não serem considerados como grupos de risco.

Considera-se que a frequência dos registros depende da condição do paciente. Nessa perspectiva, quanto maior a gravidade, complexidade e instabilidade física e emocional, maior deve ser a frequência dos registros na $\mathrm{PE}^{(9)}$, associada à adequação das mesmas às necessidades do paciente.

Outro item relevante no desempenho das $\mathrm{PE}$ se relacionou às Prescrições incompletas cuja incidência foi maior para a falta de identificação do profissional que a prescreveu, seja pela ausência de carimbo ou nome do enfermeiro.

A problemática das PE incompletas corrobora com os resultados de outro estudo brasileiro que constatou falta de identificação do profissional de enfermagem responsável pela Prescrição ${ }^{(3)}$. A falta de assinatura nas PE infringe os padrões de qualidade, pois a identificação do prescritor enfermeiro, além de uma questão ética, é um dos itens de observação do Manual Brasileiro de Acreditação Hospitalar e também uma exigência legal do Conselho Federal de Enfermagem ${ }^{(20)}$.

No enfoque da exatidão das PE, destaca-se que essa deve ser realizada pelo enfermeiro de forma ordenada e pautada em conhecimento científico, para que atue no sentido de atender as necessidades do paciente. Com base nisso, a inadequação ou não realização da $\mathrm{PE}$, tende a prejudicar, em primeira instância, o cliente, a atuação do profissional e de toda a sua equipe.

Ressaltam-se as diferenças significativas $(\alpha<0,05)$ para os resultados acerca das PE incompletas 
entre os dois hospitais (0,00) e nesse enfoque faz-se pertinente destacar que o hospital 1 possui 72 leitos e em média 1,5 enfermeiro para cada 15 leitos, considerando-se a presença do enfermeiro voluntário nos dois plantões diurnos. Já o Hospital 2 possui 316 leitos e 02 enfermeiros para cada 18 leitos. Dados desse tipo, relacionados ao dimensionamento da equipe, a carga de trabalho e às normas administrativas, necessitam ser investigados, pois certamente interferem na realização do Processo de Enfermagem ${ }^{(1)}$.

Na Tabela 2, onde constam os dados referentes aos prontuários que apresentaram ou não a $\mathrm{PE}$, se observa que dos 1307 prontuários analisados, 18 (1,37\%) não apresentavam PE. Esse dado é preocupante porque a ausência de $\mathrm{PE}$ pode resultar em consequências negativas para o cuidado, haja vista que além de comprometer a valorização e o reconhecimento do papel do enfermeiro, coloca em risco a assistência adequada ao paciente ${ }^{(18)}$.

Quanto aos dados referentes à ausência de PE, encontrou-se diferença significativa $(\alpha<0,05)$ entre as instituições $(0,02)$. Nesse sentido, o hospital 1 apresentou maior taxa de ausência de PE (Tabela 2).

Ressalta-se o fato deste estudo ter sido realizado em hospitais de ensino os quais apresentam certas particularidades que dificultam a assistência de enfermagem. Nesse caso, os enfermeiros, além das atividades assistenciais, muitas vezes, atuam no campo do ensino e da pesquisa, podendo ser esta a causa pela qual as instituições 1 e 2 apresentaram 2,67\% e 0,85\% de ausência de PE, respectivamente.

A influência das características institucionais para o desenvolvimento do Processo de Enfermagem e da PE foi abordado por autores brasileiros ${ }^{(5,13)}$ que se referem às dificuldades à sua aplicação nos hospitais universitários. De acordo com esses autores, tais empecilhos estão atrelados à alta carga de trabalho, à falta de dimensionamento adequado da equipe e à dificuldade do enfermeiro em atuar no campo da assistência e do ensino.

No que diz respeito à influência do meio para a realização da PE, faz-se pertinente destacar o papel do enfermeiro, no entanto ele não pode ser responsabilizado como único ponto causal para o sucesso ou fracasso da PE, porque outros fatores podem intervir no processo assistencial como: a gravidade do paciente e as características organizacionais ${ }^{(5,14)}$.

Quanto à oportunização de meios para o desenvolvimento do Processo de Enfermagem de qualidade, é imprescindível que haja adequações gerenciais por parte das instituições no sentido de propiciar e instrumentalizar os enfermeiros para o desempenho da PE, por meio de cursos de capacitação e normas organizacionais que possibilitem realizá-las adequadamente ${ }^{(5)}$.

Mediante a importância das PE para um cuidado de qualidade, livre de iatrogenias e erros, a avaliação das PE é fundamental como estratégia diagnóstica e como forma de readequação dos cuidados $_{\text {prescritos }}^{(1)}$.

Perante a necessidade de avaliação dos padrões de qualidade da PE, conforme a abordagem de Cianciarullo, Fugulin e Andreoni ${ }^{(18)}$ (Tabela 3), é possível constatar que $75,4 \%$ e $85,85 \%$ das $\mathrm{PE}$ foram consideradas adequadas para os hospitais 1 e 2 respectivamente. Contudo, chama atenção os percentuais de preenchimento incompleto, não preenchimento e preenchimento incorreto. Principalmente neste último, os hospitais 1 e 2 obtiveram escores de 12,56\% e 11,46\% respectivamente. Esses dados nos fazem pensar que são necessários investimentos urgentes por parte das gerências destas instituições no sentido de capacitar os enfermeiros das unidades avaliadas para que apreciem de forma correta e completa as necessidades dos pacientes sob a sua responsabilidade.

Ao analisar separadamente cada quesito das $\mathrm{PE}$, se observa que alguns itens, alcançaram padrões de qualidade nas duas instituições, como é o caso das 1083 (82,8\%) PE com elaboração correta; $52(3,97 \%)$ com preenchimento incompleto e as 18 (1,37\%) ausentes (não preenchidas).

Os resultados de inadequação das PE aos padrões de qualidade também foram abordados em uma pesquisa brasileira ${ }^{(5)}$ durante a implementação do Processo de Enfermagem em uma unidade crítica. Nesse estudo, os sujeitos (enfermeiros) indicaram dificuldades no desenvolvimento da PE devido à falta de capacitação profissional.

Faz-se mister aludir que, uma das principais dificuldades observadas no cenário atual da enfermagem quanto à prática da $\mathrm{PE}$ ainda se relaciona à falta de capacitação profissional, pois muitos enfermeiros não possuem embasamento teórico-prático para a implementação do Processo de Enfermagem, em especial, à identificação dos diagnósticos de enfermagem e elaboração da $\mathrm{PE}^{(5,14)}$. Nessa perspectiva, a Prescrição, por depender do conhecimento e da habilidade do enfermeiro em realizar o Processo de Enfermagem, necessita de mais investimentos, pois, a realização equivocada 
ou ausente desta etapa pode comprometer não só a recuperação, mas a segurança e a saúde do cliente como um todo ${ }^{(8)}$.

Os resultados deste estudo apontam à necessidade do enfermeiro e das lideranças que atuam no processo de cuidado reavaliar a sua prática com escopo de assumir sistematicamente o Processo de Enfermagem e com isso, a PE como instrumento que atua em favor da enfermagem e da qualidade do seu trabalho (cuidado).

\section{CONCLUSÕES}

Esta pesquisa constatou que, apesar de $1.083(82,8 \%)$ das PE analisadas estarem corretas e adequadas, 52 (3,97\%) apresentaram preenchimento incompleto e 18 (1,37\%) não foram preenchidas, o que sinaliza para o não atendimento a todos os padrões de qualidade propostos nesse estudo.

No que se refere à importância da $\mathrm{PE}$ para a qualidade do cuidado, os resultados deste estudo possibilitam refletir acerca da prática do enfermeiro no processo de atendimento à saúde e apontam para a existência de lacuna entre a teoria e a prática deste profissional. Mediante a esse fato, torna-se imprescindível, tanto no nível acadêmico como no âmbito profissional, discutir e repensar o papel do profissional enfermeiro no gerenciamento do cuidado à saúde, bem como, contextualizar sobre as dificuldades na elaboração e implementação da PE.

A avaliação da PE se constitui em estratégia para a obtenção de dados diagnósticos que possibilitam a sua readequação e aprimoramento, com vistas à melhoria da prática profissional do enfermeiro e à qualidade do cuidado. Com isso, apesar da sua importância, este estudo comporta limitações que podem e devem ser elucidadas por meio de investigações futuras, que tenham como foco as reais causas das inadequações e/ ou falta de PE.

Considera-se que os achados desse estudo poderão ser utilizados por enfermeiros, pesquisadores e chefias de instituições de saúde, no sentido de desencadear discussões e ações voltadas à melhoria dos padrões de qualidade das PE.

Conclui-se que nos hospitais investigados a maioria da $\mathrm{PE}$ atende aos requisitos propostos por Manganaro ${ }^{(16)}$, entretanto faz-se necessário promover ações com vistas à melhoria das $\mathrm{PE}$, sejam no aspecto qualitativo, como no quantitativo.

\section{REFERÊNCIAS}

1 Tronchin DMR, Meleiro MM, Takahashi. A qualidade e a avaliação dos serviços de saúde e de enfermagem. In: Kurcgant P, Tronchin DMR, Fugulin FMT, Peres HC, Massarollo MCKB, Fernandes MFP, et al. Gerenciamento em Enfermagem. Rio de Janeiro: Guanabara Koogan; 2010. p. 75-88.

2 Donabedian A. The definition of quality and approaches to its assessment. Ann Arbor: Health Adminstration Press; 1980.

3 Setz V, D’Innocenzo M, Avaliação da qualidade dos registros de enfermagem no prontuário por meio da auditoria. Acta Paul Enferm. 2009; 22(3):313-7.

4 Mezomo JC. Gestão da qualidade na saúde: princípios básicos. Barueri: Manole; 2001.

5 Sentone ADD. Análise dos indicadores da qualidade na elaboração da prescrição de enfermagem em uma unidade de internação de um hospital universitário público [dissertação]. Ribeirão Preto: Escola de Enfermagem de Ribeirão Preto, Universidade de São Paulo; 2005.

6 Venturini DA, Matsuda LM, Waidman MAP. Produção científica brasileira sobre sistematização da assistência de enfermagem. Ci Cuidado Saúde. 2009; $8(4): 707-715$.

7 Carpenito M, Lynda J. Manual de diagnósticos de enfermagem. Porto Alegre: Artmed; 2008.

8 Keenan GM, Yakel E, Tschannen T, Mandeville M. Documentation and the Nurse Care Planning Process. In: Hughes RG, editor. Patient Safety and Quality: An Evidence-Based Handbook for Nurses [Internet]. Rockville: Agency for Healthcare Research and Quality; 2008 [citado 2011 nov 28]. Disponível em: http://www. ncbi.nlm.nih.gov/books/NBK2674/?report=printable

9 College of Registered Nurses of British Columbia. Practice Support: nursing documentation. Vancouver: CRNBC; 2007. Disponível em:< https://www.crnbc. $\mathrm{ca} /$ Standards/Lists/StandardResources/151Nursin gDocumentation.pdf >. Acesso em 28 Nov. 2011.

10 Almeida MA, Seganfredo DH, Canto DF, Menna Barreto LN. Aplicabilidade da classificação dos resultados de enfermagem em pacientes com déficit no autocuidado: banho/higiene. Rev Gaúcha Enferm. 2010; 31(1):33-40.

11 Tannure MC, Gonçalves AMP. SAE, Sistematização da Assistência de Enfermagem. Rio de Janeiro: Guanabara Koogan; 2009. 
12 Roque KE, Prates EC, Tonini MT. Pós-operatório de transplante renal: avaliando o cuidado e o registro do cuidado de enfermagem. Esc Anna Nery. 2007; 11(3): $409-16$.

13 Repeto AM, Souza MF. Avaliação da realização e do registro da Sistematização da Assistência de enfermagem (SAE) em um hospital universitário. Rev Bras Enferm. 2005; 58(3):325-9.

14 Pokorski S, Moraes MA, Chiarelli R, Costanzi AP, Rabelo ER. Processo de enfermagem: da literatura à prática. O quê de fato nós estamos fazendo? Rev. Latino-Am. Enfermagem [Internet]. 2009 [citado 2010 nov 28]; 17(3):302-307. Disponível em: http://www.scielo.br/scielo.php? script=sci_ arttext\&pid=So 104-1 1692009000300004\&lng=en \&nrm=iso >. ISSN 0104-1 169. doi: 10.1590/So10411692009000300004 .

15 Takahashi AA, Barros ALBL, Michel JLM, Souza MF. Dificuldades e facilidades apontadas por enfermeiras de um hospital de ensino na execução do processo de enfermagem. Acta Paul Enferm. 2008; 21(1): 32-8.
16 Manganaro MM. Sistematização da Assistência de Enfermagem. In: Murta GF. Saberes e Práticas: Guia para Ensino e aprendizagem de enfermagem. São Caetano do Sul: Difusão Editora; 2006. p.22 1-242.

17 Vituri DW. Desenvolvimento e validação de um instrumento para avaliação da qualidade do cuidado de enfermagem. 2007. [dissertação]. Maringá (PR): Departamento de Enfermagem da Universidade Estadual de Maringá; 2007.

18 Cianciarullo TI, Fugulin FMT, Andreoni S. O processo de cuidar. In: Cianciarullo TI, Fugulin FMT, Andreoni S. A hemodiálise em questão: opção pela qualidade assistencial. C\&Q. São Paulo: Ícone; 1998. p. $37-50$.

19 Ministério da Saúde (BR), Conselho Nacional de Saúde. Resolução 196, de 10 de outubro de 1996: diretrizes e normas regulamentadoras de pesquisa envolvendo seres humanos. Brasília (DF); 1996.

20 Labbadia LL, Adami NP. Avaliação das anotações de enfermagem em prontuários de um hospital universitário. Acta Paul Enferm. 2004;17(1):55-62.

\author{
Endereço do autor / Dirección del autor / \\ Author's address: \\ Gelena Lucineia Gomes da Silva Versa \\ Rua Visconde do Rio Branco, 3511 , Centro \\ 85810-180, Cascavel, PR \\ E-mail: gelenaenfermagem@yahoo.com.br
}

